\title{
The origin of GEMS in IDPs as deduced from microstructural evolution of amorphous silicates with annealing
}

\author{
C. Davoisne ${ }^{1}$, Z. Djouadi ${ }^{2}$, H. Leroux ${ }^{1}$, L. d'Hendecourt ${ }^{2}$, A. Jones ${ }^{2}$, and D. Deboffle ${ }^{2}$ \\ ${ }^{1}$ Laboratoire de Structure et Propriétés de l'Etat Solide, UMR 8008, Université des Sciences et Technologies de Lille, \\ 59655 Villeneuve d'Ascq Cedex, France \\ e-mail: hugues.leroux@univ-lille1.fr \\ 2 Institut d'Astrophysique Spatiale (IAS), Bâtiment 121, Université Paris-Sud 11, CNRS (UMR 8617), 91405 Orsay, France
}

Received 14 December 2005 / Accepted 8 January 2006

\section{ABSTRACT}

\begin{abstract}
Aims. We present laboratory studies of the micro-structural evolution of an amorphous ferro-magnesian silicate, of olivine composition, following thermal annealing under vacuum.

Methods. The amorphous silicate was prepared as a thin film on a diamond substrate. Annealing under vacuum was performed at temperatures ranging from 870 to $1020 \mathrm{~K}$. After annealing the thin films were extracted from the substrate and analysed by transmission electron microscopy to infer their microstructural and compositional evolution.

Results. Spheroidal metallic nano-particles $(2-50 \mathrm{~nm})$ are found within the silicate films, which are still amorphous after annealing at $870 \mathrm{~K}$ and partially crystallized into forsterite for annealing up to $1020 \mathrm{~K}$. We interpret this microstructure in terms of a reduction of the initial amorphous silicate $\mathrm{FeO}$ component, because of the carbon-rich partial pressure in the furnace due to pumping mechanism. Annealing in a controlled oxygen-rich atmosphere confirms this interpretation.

Conclusions. The observed microstructures closely resemble those of the GEMS (Glass with Embedded Metal and Sulphides) found in chondritic IDPs (Interplanetary Dust Particles). Since IDPs contain abundant carbonaceous matter, a solid-state reduction reaction may have occurred during heating in the hot inner regions of the proto-solar disc. Related to this, the presence of forsterite grains grown from the amorphous precursor material clearly demonstrates that condensation from gaseous species is not required to explain the occurrence of forsterite around young protostars and in comets. Forsterite grains in these environments can be formed directly in the solid phase by thermal annealing of amorphous ferro-magnesian silicates precursor under reducing conditions. Finally, locking iron as metallic particles within the silicates explains why astronomical silicates always appear observationally Mg-rich.
\end{abstract}

Key words. methods: laboratory - techniques: microscopy - ISM: dust, extinction

\section{Introduction}

Interplanetary dust particles (IDPs) are probably the most pristine materials in the Solar System. The mineralogy of IDPs could therefore yield critical information about the processes that occurred in the solar nebula and the early solar system. IDPs are a fine-grained mixture of materials. Among them, GEMS (Glass with Embedded Metal and Sulphides) are a major component of the anhydrous IDPs (Bradley 1994). GEMS are typically a few $100 \mathrm{~nm}$ in size and are composed of a silicate glass which includes small (typically 10-50 nm-sized) and rounded grains of $\mathrm{Fe}, \mathrm{Ni}$ metal and sulphide. It has been proposed that the amorphous character of GEMS originates from pre-accretional irradiation effect (Bradley 1994). Some GEMS also contain some crystalline silicate inclusions of predominantly forsterite (e.g., Bradley 2003). Some fraction of the GEMS is believed to be of interstellar origin. Their infrared spectra compare well with the spectra of the silicate grains in molecular clouds, comets and Herbig Ae/Be stars
(Bradley et al. 1999). Some GEMS display oxygen isotopic anomalies, indicating a pre-solar origin (Messenger et al. 2003; Floss et al. 2005). GEMS with solar oxygen isotopic compositions could either have been formed in the early Solar System or have been homogenized through processing in the interstellar medium (Tielens 2003). Another interesting feature of GEMS is that their bulk elemental compositions are frequently enriched in $\mathrm{SiO}_{2}$, as shown by the relatively low $\mathrm{S} / \mathrm{Si}, \mathrm{Mg} / \mathrm{Si}$, $\mathrm{Ca} / \mathrm{Si}$ and $\mathrm{Fe} / \mathrm{Si}$ ratios, however, the $\mathrm{Al} / \mathrm{Si}$ ratio is found to be approximately chondritic (Schramm et al. 1989; Keller \& Messenger 2004). Finally, it should be mentioned that GEMS are frequently found enclosed by, or are in close association with, carbonaceous materials (e.g., Thomas et al. 1993; Bradley et al. 1999; Keller et al. 2000; Keller et al. 2004). Despite numerous studies devoted to the GEMS in IDPs there is currently no consensus regarding their origin and their formation process. Some GEMS appear to have a clear pre-solar origin, while others have a solar isotopic composition, which could be the result of homogenization in the interstellar medium or of 
formation in the solar system (Messenger et al. 2003). An interstellar, shock-accelerated dust origin for these GEMS has recently been proposed (Westphal \& Bradley 2004). Alternatively it was also proposed that GEMS which contain relict crystalline grains could be pseudomorphs formed by irradiation processing of crystals free-floating in space (Bradley \& Dai 2004). The amorphous state of the GEMS would therefore appear to favor an interstellar origin. Indeed interstellar silicates are believed to be largely amorphous (Mathis 1990; Demyk et al. 1999; Kemper et al. 2004), an observation that can be explained by low-energy irradiation in supernova-generated, interstellar shock waves (Demyk et al. 2001; Carrez et al. 2002). Under this shock wave irradiation hypothesis the amorphous interstellar silicates grains could have been progressively heated during their incorporation into, and their transport through, the proto-planetary discs. In case of extreme heating they would progressively undergo annealing, solid-state transformation, melting and volatilization. Due to solar wind and/or turbulence in the proto-stellar disc, a fraction of the amorphous silicate particles should have escaped the severe heating processes.

In this paper we examine the possibility that GEMS could have been formed by the heating of precursor, amorphous, interstellar grains in the solar nebula. In our experiments, aimed at understanding the origin of the GEMS, thin (50-100 nm thick) films of amorphous silicates, of olivine composition, were annealed under vacuum at 870,970 and $1020 \mathrm{~K}$ on a diamond substrate. The microstructure was investigated using a transmission electron microscopy (TEM) and X-ray energy dispersive spectroscopy (EDS) set-up attached to the TEM.

\section{Experiments}

Interstellar silicates are small grains (typically $10-500 \mathrm{~nm}$ diameter) and have a high surface to volume ratio. We therefore opted to work with thin films of amorphous silicates (50-100 nm thick) of olivine composition. The starting sample was a San-Carlos (Arizona) olivine $\mathrm{Mg}_{1.8} \mathrm{Fe}_{0.19} \mathrm{Ni}_{0.01} \mathrm{SiO}_{4}$. The amorphous films were prepared by a standard electronbeam evaporation technique. A current was applied through a tungsten filament in the vacuum chamber until the thermionic emission of electrons occurs. By means of electromagnets the electrons are focussed onto the surface of the sample in a tungsten crucible. The electron density is sufficient to heat the sample, partially melt it and form a vapour that condensed on the substrate. The substrate used in our experiment was a $3 \mathrm{~mm}$ diameter diamond disc. This substrate was chosen for several reasons. Firstly, diamond has an IR signature far from the absorption regions of silicates (i.e. $\sim 10$ and $20 \mu \mathrm{m}$ ) allowing thus the IR characterization of the thin silicate films and their comparison with data from the Infrared Space Observatory or the Spitzer Space Telescope (Djouadi et al. 2005). Secondly, diamond substrates have smooth surfaces without significant roughness. This makes for an easy extraction of the thin film for TEM characterization. After their synthesis the samples were placed in a furnace and were annealed under vacuum at 870 , $970 \mathrm{~K}$ and $1020 \mathrm{~K}$, at low pressure $\left(10^{-10} \mathrm{bar}\right)$. In order to test the influence of oxygen fugacity, some experiments were also conducted within an $\mathrm{O}_{2}$ gas circulation system, at low pressure $\left(10^{-7}\right.$ bar $)$. After annealing some areas of the film were scraped from the substrate and deposited on a carboncoated TEM foil. The TEM micro-structural observations were carried out with a Philips CM30 TEM operating at $300 \mathrm{kV}$. The crystallographic characterization was achieved using selected area electron diffraction (SAED). The microscope was also equipped with an X-ray energy dispersive spectrometer (EDS) for microanalysis.

\section{Results}

After deposition on the diamond substrate, and removal for TEM characterization, the thin film is completely amorphous. EDS measurements show that the composition is close to the starting San-Carlos olivine, but a slight $\mathrm{SiO}_{2}$ enrichment is noted. For the annealing experiment at $870 \mathrm{~K}$, the silicate film remains amorphous, even for a long duration (up to $780 \mathrm{~h}$ ). For annealing at 970 and $1020 \mathrm{~K}$ (respectively for 55 and $3 \mathrm{~h}$ ), partial crystallization is observed by IR spectroscopic analysis. The microscopic investigation we present concern only the samples before their total crystallization. The main characteristic of all the samples annealed (at $10^{-10}$ bar and without $\mathrm{O}_{2}$ circulation) is the presence of widespread iron-nickel nano-particles (Fig. 1) randomly distributed, 2-50 $\mathrm{nm}$ in size, for which the compositions are highly variable, from 3 to $50 \% \mathrm{Ni}$. The amorphous phase which encloses the metallic globules is free of Fe. This microstructure and microanalyses clearly show that iron, initially in form of $\mathrm{FeO}$, has segregated from the amorphous phase in the form of metallic precipitates. Despite the presence of metallic precipitates, the average composition (amorphous silicate + metallic nano-particules) is found strongly depleted in $\mathrm{Fe}$. A moderate loss of $\mathrm{Mg}$ is also observed.

The films annealed at 970 and $1020 \mathrm{~K}$ contain some forsterite crystals (Fig. 2). Their grain size ranges from 50 to $250 \mathrm{~nm}$. They are pure forsterite, i.e., they are iron free. The forsterite grain abundance varies with the analyzed region, with some areas free of them, while others make up about $50 \%$ of the surface of the thin film. The forsterite crystal morphologies are dendritic, as shown by the irregular growth interface in contact with the remaining amorphous material. The absence of anhedral morphologies is compatible with annealing at moderate temperatures.

Finally, in the samples annealed at higher oxygen pressure $\left(10^{-7}\right.$ bar $)$, the amorphous phase of the thin films contain no metallic precipitates. This comparative experiment shows that the composition of the residual atmosphere in our vacuum system plays a crucial role in microstructure development, in particular when the surface to volume ratio is high as in our thin films.

\section{Discussion}

In this work we have attempted to simulate the incorporation of amorphous interstellar silicates into the hot inner disc of a proto-star. We have thus thermally-annealed amorphous ferromagnesian silicates under vacuum. The main objective was to compare the mineralogies obtained in our experiment with 


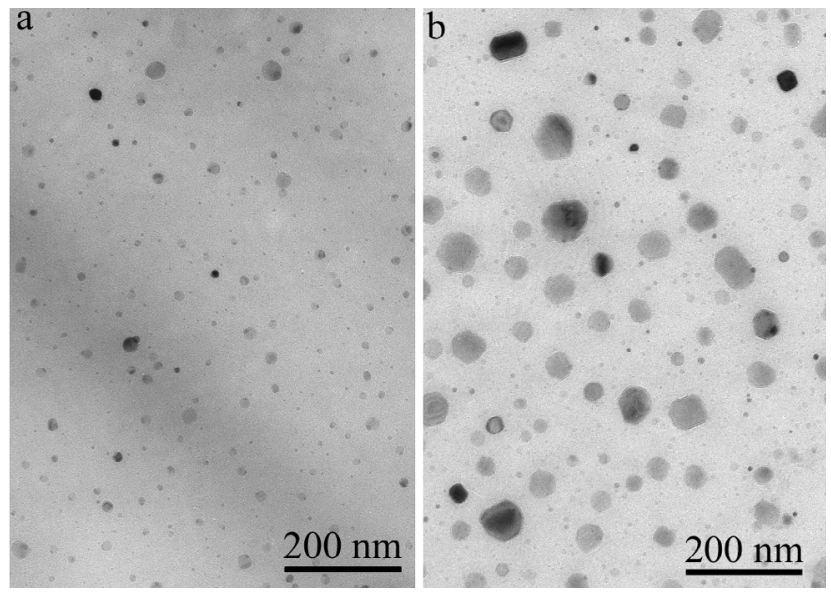

Fig. 1. TEM micrograph of annealed sample a) at $870 \mathrm{~K}$ for $780 \mathrm{~h}$ and b) at $1020 \mathrm{~K}$ for $3 \mathrm{~h}$. Rounded metallic nano-particles enclosed in the amorphous silicate. They formed by a reduction reaction and further precipitation since metallic $\mathrm{Fe}$ is immiscible in silicates. The microstructure closely ressembles to those to GEMS found in IDPs.

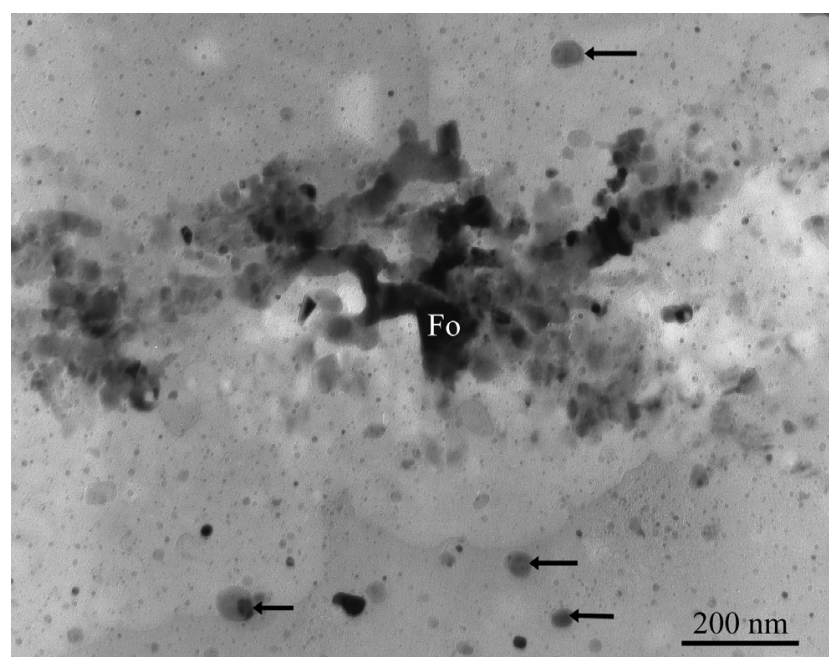

Fig. 2. TEM micrograph of sample annealed at $970 \mathrm{~K}$ (55 h) showing a forsterite crystal (Fo) embedded in a amorphous matrix. Note the dentritic structure at the edge of the grains. Some metal particles are also present in the amorphous phase (some of them are arrowed).

those of the IDPs, in particular the GEMS, some of which are suspected of having an interstellar origin (Bradley 1994; Bradley et al. 1999; Messenger et al. 2003). The main findings of our study are that the annealing of amorphous ferromagnesian silicates leads to the formation of forsterite crystals and metallic iron within a residual amorphous phase. Since the iron in the starting material was in the form of $\mathrm{FeO}$, the formation of metallic particles clearly shows that reduction has occurred according to the reaction: $\mathrm{FeO} \rightarrow \mathrm{Fe}+1 / 2 \mathrm{O}_{2}$. The thermodynamical data associated with this reaction indicate that the equilibrium oxygen partial pressure is of the order of $10^{-20}$ bar at $1000 \mathrm{~K}$ (Matas et al. 2000). The total pressure during annealing was $10^{-10} \mathrm{bar}$. The very low oxygen partial pressure $\left(<10^{-20}\right.$ bar $)$ necessary for the reduction reaction to proceed is probably due to carbon-rich contaminants coming from the pumping system, which consumes oxygen by the reaction $\mathrm{C}+1 / 2 \mathrm{O}_{2} \longleftrightarrow \mathrm{CO}$ and thus induces metal formation according to the reaction $\mathrm{FeO}+\mathrm{C} \rightarrow \mathrm{Fe}+\mathrm{CO}$. This hypothesis is confirmed by another annealing experiment performed at higher $\mathrm{O}_{2}$ vapor pressure by injecting an oxygen flux at controlled pressure of $10^{-7}$ bar. In this configuration, the carbonrich species coming from the vacuum pumps react with the $\mathrm{O}_{2}$ gas within the furnace, preventing the formation of metallic iron on the sample surface.

The presence of iron precipitates within a silicate glass closely resembles the microstructure observed within the GEMS (e.g., Bradley 1994). Since IDPs contain a large carbonaceous material fraction, we suggest that metal formation in GEMS could result from a reduction reaction during the annealing of amorphous, ferro-magnesian precursor silicates at temperatures below $1000 \mathrm{~K}$. This annealing could have occurred within the hot, inner regions of the proto-solar disc. The occurrence of forsterite crystals in GEMS is clear (Bradley 2003) and our experiments show that this occurrence can be explained by an in situ crystallization of an amorphous, ferromagnesian silicate precursor under reducing conditions. This formation mechanism is also compatible with the detection of pre-solar (non-solar oxygen isotopic composition) forsterite crystals in IDPs (Messenger et al. 2003) and presolar olivines and GEMS-like grains in primitive meteorites (Mostefaoui \& Hoppe 2004). Indeed, the isotopic composition of the forsterite and olivine grains appears to be close to that of the precursor materials from which they were formed, i.e., for some of them presolar amorphous silicate grains. Forsterite grains are found in cometary grains (e.g., Crovisier et al. 1997; Wooden et al. 1999, 2000), around young stars (e.g., Waelkens et al. 1996; Malfait et al. 1998) and also around evolved stars (Waters et al. 1996), as revealed by ISO observations. In contrast, interstellar silicates are thought to be almost completely amorphous (Li \& Draine 2001; Kemper et al. 2004), showing that crystalline grains are formed exclusively within circumstellar environments. Thus, the properties of the crystalline grains (abundance, chemical composition, grain size, etc.) could provide essential information on the conditions of their formation and their subsequent evolution in discs. For instance, the crystalline silicates in comets provide an interesting illustration of this. It has been suggested that crystallization of the amorphous precursors occurred prior to comet formation, probably in the hot, inner-edge regions of the accretion disc followed by radial transport and mixing into the comet-forming region (e.g., Bockelée-Morvan et al. 2002).

In addition to the spectroscopic observations, another source of information is the study of primitive solar system materials available for laboratory characterization. For example, the presence of Mg-rich, crystalline silicates is restricted to very primitive materials, such as micrometeorites and IDPs (Bradley 1983; Bradley et al. 1992; Bradley 2003) and, to date, one meteorite (Mostefaoui \& Hoppe 2004). Two models are frequently invoked to explain the presence of crystalline $\mathrm{Mg}$-silicates within circumstellar environments and in primitive materials. Firstly, they can be formed via gas-phase condensation. The equilibrium condensation sequence shows that pure $\mathrm{Mg}$ silicates appear first, as predicted in theoretical models (e.g., Grossman 1972), and as found in experimental condensation experiments (Rietmeijer et al. 1999). Another way 
to form crystalline silicates is by the thermal annealing of amorphous precursors, such as amorphous, interstellar silicate grains. In this hypothesis, the formation of $\mathrm{Mg}$-rich silicates is not obvious, although it has been shown that iron silicates require higher temperatures to crystallize as compared to magnesium silicates (e.g., Hallenbeck et al. 2000). Our experimental study shows that forsterite grains can indeed be formed from amorphous precursors at relatively moderate temperatures (i.e., around $1000 \mathrm{~K}$ ) in the presence of carbon-rich species. This assemblage (silicate+carbonaceous matter) is typical of the inferred interstellar grain composition and according to the silicate core-organic refractory mantle model (e.g., Greenberg \& Li 1997). High temperature gas-phase condensation is thus not exclusively required to explain the presence of crystalline and magnesium-rich silicates in circumstellar environments.

\section{Conclusion}

We have performed thermal annealing experiments under vacuum at 870,970 and $1020 \mathrm{~K}$ on an amorphous, ferromagnesian, silicate thin film on diamond substrate. The annealed samples were studied by transmission electron microscopy and energy dispersive spectroscopy. The main results are the occurrence of spheroidal metallic particles and the formation of pure forsterite crystals. The overall microstructures of our annealed samples closely resemble those found in the GEMS component of the chondritic IDPs. We interpret the formation of the metallic spheroids, and the accompanying forsterite crystals, in terms of a reduction of the initial $\mathrm{FeO}$ component by carbon from the reduced atmosphere of our experimental setup. Given that IDPs contain abundant carbonaceous matter, we propose that solid-state annealing in association with a reduction reaction at relatively moderate temperatures (below $1000 \mathrm{~K}$ ), has occurred during the heating of amorphous, pre-solar/interstellar, silicate grains in the hot inner-disc regions of the proto-planetary solar nebula. Subsequently, this material could have been incorporated into the outer, cooler and comet-forming regions of the nebula through radial transport and mixing. Such a process explains also the occurrence of crystalline $\mathrm{Mg}$-rich silicates observed in comets, the iron being locked in a metallic nanophase and thus becoming unobservable.

Acknowledgements. The authors would like to thank the French INSU-CNRS "programme national de planétologie, PNP" for partial financial support of this work.

\section{References}

Bockelée-Morvan, D., Gautier, D., Hersant, F., Huré, J. M., \& Robert, F. 2002, A\&A, 384, 1107
Bradley, J. P. 1994, Science, 265, 925

Bradley, J. P. 2003, The Astromineralogy of Interplanetary Dust Particles, in Astrominerlogy, ed. T. Henning (Berlin, Heidelberg: Springer-Verlag), LNP, 609, 217

Bradley, J. P., \& Dai, Z. R. 2004, ApJ, 617, 650

Bradley, J. P., Brownlee, D. E., \& Veblen, D. R. 1983, Nature, 301, 473

Bradley, J. P., Humecki, H. J., \& Germani, M. S. 1992, ApJ, 394, 643

Bradley, J. P., Keller, L. P., Snow, T. P., et al. 1999, Science, 285, 1716

Carrez, P., Demyk, K., Cordier, P., et al. 2002, Meteoritics Planet. Sci., 37, 1599

Crovisier, J., Leech, K., Bockelée-Morvan, D., et al. 1997, Science, 275,190

Demyk, K., Jones, A. P., Dartois, E., Cox, P., \& d'Hendecourt, L. 1999, A\&A, 349, L267

Demyk, K., Carrez, P., Leroux, H., et al. 2001, A\&A, 368, L38

Djouadi, Z., d'Hendecourt, L., Leroux, H., et al. 2005, A\&A, 440, 179

Floss, C., Stadermann, F. J., Bradley, J. P., et al. 2004, Science, 303, 1355

Greenberg, J. M., \& Li, A. 1997, Adv. Space Res., 19, 981

Grossman, L. 1972, Geochim. Cosmochim. Acta, 36, 597

Hallenbeck, S. L., Nuth, J. A., \& Nelson, R. N. 2000, ApJ, 535, 247

Keller, L. P., \& Messenger, S. 2004, Lunar Planet. Sci. Conf., 35, 1985

Keller, L. P., Messenger, S., \& Bradley, J. P. 2000, J. Geophys. Res., 105, 10397

Keller, L. P., Messenger, S., Flynn, G. J., et al. 2004, Geochim. Cosmochim. Acta, 68, 2577

Kemper, F., Vriend, W. G., \& Tielens, A. G. G. M. 2004, ApJ, 609, 826

Li, A., \& Draine, B. T. 2001, ApJ, 550, L213

Malfait, K., Waelkens, C., Waters, L. B. F. M., et al. 1998, A\&A, 332, L25

Matas, J., Ricard, Y., Lemelle, L., \& Guyot, F. 2000, Contrib. Miner. Petrol, 140, 73

Mathis, J. S. 1990, ARA\&A, 28, 37

Messenger, S., Keller, L. P., Sterdermann, F. J., Walker, R. M., \& Zinner, E. 2003, Science, 300, 105

Mostefaoui, S., \& Hoppe, P. 2004, ApJ, 613, L149

Rietmeijer, F. J. M., Nuth, J. A., \& Karner, J. M. 1999, ApJ, 527, 395

Schramm, L. S., Brownlee, D. E., \& Wheelock, M. M. 1989, Meteoritics, 24, 99

Thomas, K. L., Blanford, G. E., Keller, L. P., Klock, W., \& McKay, D. S. 1993, Geochim. Cosmochim. Acta, 57, 1551

Tielens, A. G. G. M. 2003, Science, 300, 68

Waelkens, C., Waters, L. B. F. M., de Graauw, M. S., et al. 1996, A\&A, 315, L245

Waters, L. B. F. M., Molster, F. J., de Jong, T., et al. 1996, A\&A, 315, L361

Westphal, A. J., \& Bradley, J. P. 2004, ApJ, 617, 1131

Wooden, D. H., Harker, D. E., Woodward, C. E., et al. 1999, ApJ, 517, 1034

Wooden, D. H., Butner, H. M., Harker, D. E., \& Woodward, C. E. 2000, Icarus, 143, 126 